\title{
DIBUJAR EL TERRITORIO DE LA ARAUCANÍA, CHILE Aproximaciones cartográficas a las construcciones territoriales que se dieron desde la llegada de los españoles (siglo XVI) hasta los inicios de la República (siglo XIX)
}

\author{
Wladimir Antivil Marinao \\ Departamento de Urbanismo y Ordenación del Territorio, Universidad Politécnica de Cataluña. \\ Director de tesis: Xabier Eizaguirre Garaitagoitia. \\ Email: wladimir.mauro.antivil@estudiant.upc.edu
}

\section{RESUMEN}

El siguiente trabajo presenta un acercamiento al territorio de la Araucanía durante un espacio de tiempo (principalmente desde el siglo XVI al XIX). Durante este período el territorio fue construido por dos culturas de una forma asimétrica. Por una parte, fue construida por el pueblo mapuche o araucano y, por otra, por la colonia española. Presentaremos una exposición de las principales formas de construcción territorial que se conocen de aquella época, tanto las morfologías territoriales mapuches como las españolas. El trabajo se basa principalmente en las fuentes de la historia, la antropología y la cartografía.

Palabras clave: Morfología territorial, Historia de la Araucanía, colonización española, asentamientos mapuches.

\section{ABSTRACT}

The following work presents an approach to the territory of Araucanía during a space of time (mainly from the $16^{\text {th }}$ to the $19^{\text {th }}$ century). During this period the territory was built by two cultures in an asymmetrical way. On the one hand, it was built by the Mapuche or Araucanian people and, on the other hand, by the Spanish colony. We will present an exposition of the main forms of territorial construction that are known from that time, both the Mapuche and Spanish territorial morphologies. The work is based mainly on the sources of history, anthropology and cartography.

Keywords: Territorial morphology, History of Araucanía, Spanish colonization, Mapuche settlements. 


\section{INTRODUCCIÓN}

Estamos frente a un objeto que no es una ciudad, ni un proyecto, sino un territorio. El siguiente trabajo abarca un área de estudio extensa, lo que al día de hoy incluye tres regiones y varias provincias de Chile. A través de la información de diversas fuentes se intenta establecer algunas hipótesis de construcción de este territorio en un período de tiempo que abarca principalmente desde la conquista española (siglo XVI) hasta la primera mitad del siglo XIX. Este límite se marca, por un lado, porque desde el siglo XVI el proceso de colonización española viene a producir unas primeras transformaciones en el territorio de lo cual hay registro. Por el otro lado, se considera la primera mitad del siglo XIX, porque es el periodo justo antes en que la naciente República de Chile vendrá a colonizar definitivamente la Araucanía. Nos interesa estudiar hasta aquí porque durante la segunda mitad del siglo XIX la transformación vendrá a ser radical, con sus consecuencias hasta el día de hoy. Este territorio cuenta con bastantes trabajos de investigación, sobre todo desde algunas disciplinas como las ciencias sociales, pero no cuenta con una historia del territorio en cuanto a sus hechos físicos. En este sentido el trabajo quiere aportar con una mirada atenta a los patrones de construcción territorial mapuche y patrones de los conquistadores y la colonia española. Esto se pretende conseguir a través del dibujo y algunas fuentes escritas. Se verá como algunos elementos geográficos, como la cordillera de la Costa, fueron importantes a la hora de establecer una forma territorial, junto con eso se intentará demostrar cómo este territorio, al llegar el siglo XIX, ya poseía (aunque elementales) unas configuraciones territoriales establecidas. Se ha estudiado lo que cada pueblo o cultura acabó construyendo, sea mucho o sea poco. No hay una equivalencia en lo que cada pueblo logró construir. No son muchos los datos específicos que se tienen respecto a la construcción del territorio en este período, por esa razón el período de tiempo que se considera estudiar es bastante largo. Se ha comenzado por las construcciones que hicieron los primeros españoles, debido a que, a partir de estos se fueron desarrollando los primeros cambios del territorio, y también porque son estos los que nos dan las observaciones más antiguas que se mantienen registradas.

El trabajo tiene un carácter histórico, en el sentido de usar la historia como fuente de información, pero principalmente es un trabajo exploratorio que tiene por objeto poder dibujar los hechos territoriales, desde la disciplina de la Arquitectura, con el fin de que, pensando en su utilidad, estos puedan servir para comparaciones con el territorio más actual y ver cómo ha sido esa evolución. La aproximación, en resumen, se hace, en primer lugar, en la recopilación de autores que hablen de lo físico y territorial en esta época y, en segundo lugar, en el dibujar aquellos datos y reflexiones.

El planteamiento del dibujo descansa en el análisis de las morfologías del territorio. Esta mirada hacia el territorio proviene del Laboratorio de Urbanismo de Barcelona (1981) y trabajos de Xabier Eizaguirre (1985, 1990, 2001), donde el dibujo y la confianza en la consistencia de la forma son importantes para entender e imaginar un territorio. Estos trabajos existen bajo el supuesto que las cartografías (o dibujos del territorio), son solo una selección de una realidad territorial compleja, son una abstracción, una mirada parcial. Y esta mirada parcial es una interpretación. Hay una diferencia importante, eso sí, entre el presente trabajo y aquellos: la escala. En aquellos trabajos las escalas 1:5000 o 1:10000 permiten un tipo de observación más detallada. En nuestro caso la escala es regional, abarcando un territorio más amplio dando observaciones más generales. También ha sido de valor la mirada al territorio desde la Arquitectura dada por Vittorio Gregotti (1972)

En cuanto al origen de las fuentes es necesario aclarar algo, cuando hablamos de los mapuches lo haremos principalmente de fuentes que no provienen del propio pueblo mapuche. Prácticamente todas las fuentes provienen "del otro lado"1. Lo que consideramos son descripciones a partir de la antropología y de la historia (Latcham, 1924; Dillehay, 1990; Aldunate, 1996; Bengoa, 2008; Comisión de trabajo autónomo mapuche, 2009; Zavala, 2011). Estas fuentes, entre otras, son de valor porque permiten construir hipótesis sobre cómo se ha ido construyendo el territorio mapuche.

\section{Encuadrando el territorio}

Frecuentemente se asocia a la Araucanía $^{2}$ con el territorio que habitan y habitaron los mapuches. Si consideramos un mapa de división político administrativa actual de Chile observaremos que la Araucanía está definida claramente en una región entre región del Bío-Bío y la región del Los Ríos. Para nuestro trabajo, queremos poner en cuestionamiento aquella definición. El territorio mapuche históricamente no ha

\footnotetext{
1 La única obra, que hemos encontrado hasta, que se puede considerar desde "el lado mapuche" es la obra de Pascual Coña (2006).

2 El nombre "Araucanía" no proviene desde el propio pueblo mapuche, es un nombre, o que fue dado desde la colonia española, o desde el Estado de Chile. Es el nombre con el que se trabajará.
} 
estado sujeto a límites administrativos. Históricamente sus límites han sido difusos. Sabemos que los límites están más allá de los chilenos, llegando incluso hasta la actual Argentina. Por un asunto de orden consideraremos el territorio mapuche desde el lado chileno. Al observar diversas cartografías y descripciones hemos establecido un encuadre que inicia por el norte desde la zona cercana a la desembocadura del río Bío-Bío hasta el sur por la zona cercana a la desembocadura del río Valdivia; al poniente con el Océano Pacífico y al oriente con la cordillera de los Andes (Figura 1).

Estamos entonces frente a un territorio que no es isótropo, sino que tiende a ser largo, con una característica "cartesiana" donde la estructura de Chile coincide con el eje norte-sur y la estructura de sus ríos con el sentido este-oeste. Cuenta con la Cordillera de Los Andes que corre de norte a sur y que conforme va acercándose al sur va bajando su altura. Paralela a Los Andes está la Cordillera de la Costa, de menor altura, pero igualmente determinante a la hora de las ocupaciones mapuches y las primeras ocupaciones españolas en el territorio.

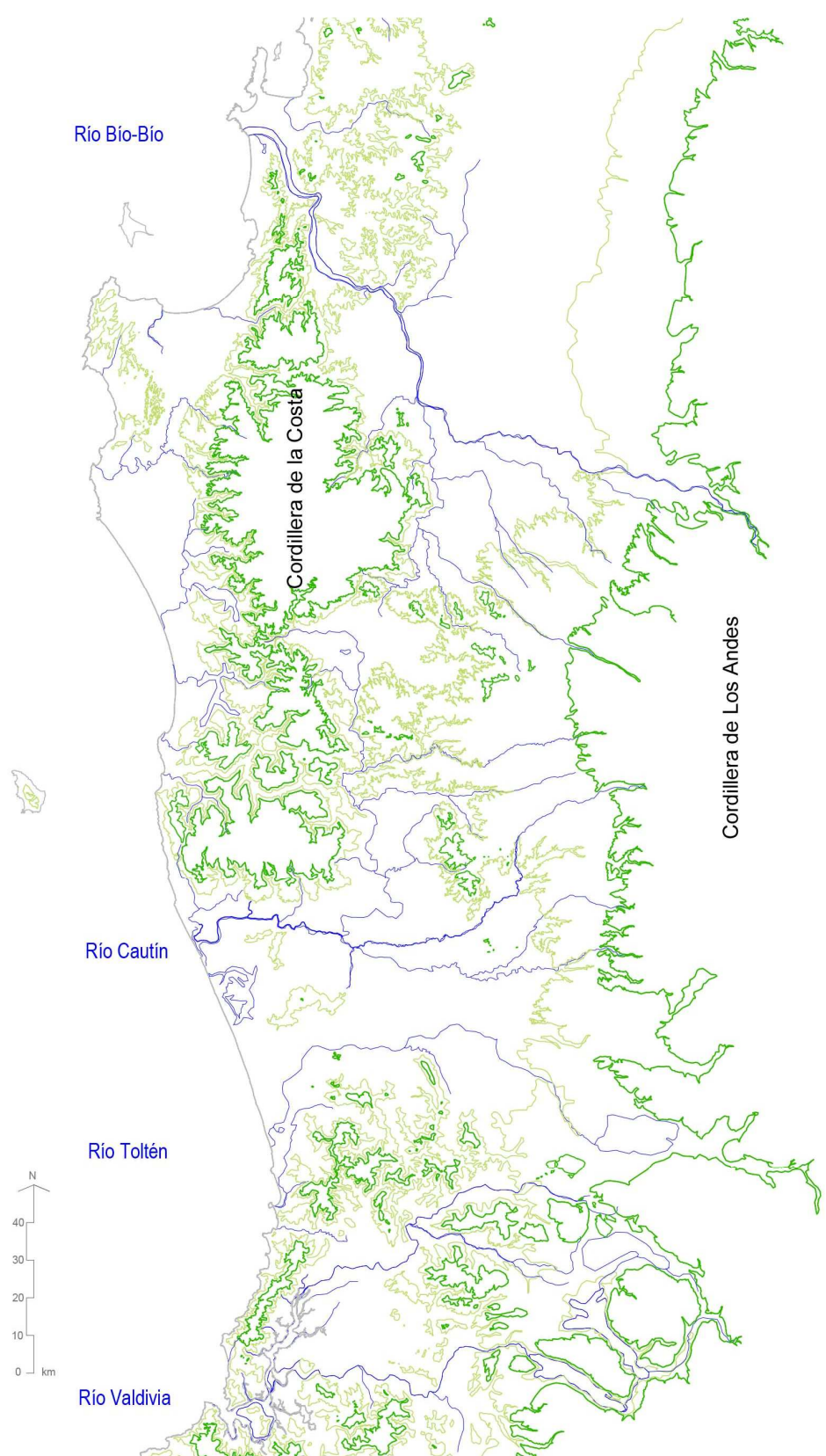

Figura 1. Encuadre escogido, señalando los límites de la Araucanía que estudiaremos. Se han dibujado los ríos importantes y dos curvas de nivel: a $200 \mathrm{~m}$ y a $400 \mathrm{~m}$ de altura. Fuente: Elaboración propia con base en cartografía del Instituto Geográfico Militar, Chile (en adelante IGM). 


\section{LA CONQUISTA Y COLONIZACIÓN ESPAÑOLA}

\subsection{La importancia de la Araucanía}

En Chile, el proceso de construcción de los primeros asentamientos urbanos comenzó en el siglo XVI, es en este momento cuando se funda la ciudad de Santiago de Chile en 1541. El conquistador de esta época es Pedro de Valdivia, quien también fue un impulsor a la hora de fundar asentamientos urbanos sobre el territorio, como expresión de poder.

Creemos que la Araucanía es importante en esta época y se puede demostrar con algunos comentarios de los cronistas. Por ejemplo, Mariño de Lobera (1865:110-111), quien define la Araucanía como los "estados de Arauco y Tucapel":

"Desde el primero dia que los españoles entraron en esta tierra de Chile siempre fué su principal intento ganar los estados de Arauco, y Tucapel por ser los mas principales de Chile, asi por la hermosura; i fertilidad de la tierra, como por la grande abundancia de oro que hai en sus minas, y aunque diversas veces lo habian intentado, como se dijo arriba, siempre se volvian ántes de llegar a donde deseaban por no ser menor la ferocidad y valentia de la jente araucana y tucapelina que su riqueza y abundancia. Y por esta causa habia puesto el gobernador tanta dilijencia en que entrase a este reino mucha jente española, teniendo siempre ante los ojos esta conquista, para lo cual juntaba siempre los mas soldados que podia. [sic]"

Si hacemos un dibujo, considerando parte de Sudamérica, veremos que los principales asentamientos españoles en la zona que corresponde hoy a Chile están agrupados en la zona de la Araucanía (Figura 2), a diferencia de Santiago de Chile o La Serena que aparecen más bien aisladas. Esto es una segunda sospecha sobre la importancia de este territorio.

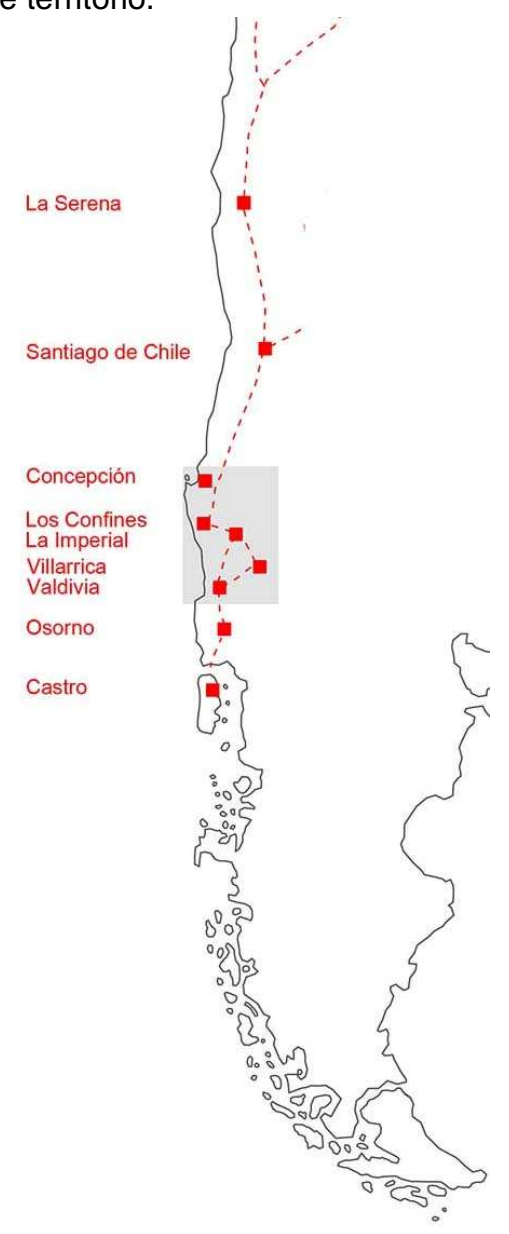

Figura 2. Principales asentamientos españoles en la zona correspondiente al Chile actual y la zona de la Araucanía que hemos encuadrado (en gris). Elaboración propia a partir de dibujo en Guarda (1978:22) quien se basa en los datos de López de Velasco. 
La primera ciudad que Pedro de Valdivia funda en Chile es La Serena situada aproximadamente a $400 \mathrm{~km}$ al norte de Santiago, la segunda ciudad que funda es la propia Santiago cuyo nombre será "Santiago del Nuevo Extremo". Una vez fundada estas ciudades Valdivia se adentra al territorio de los araucanos. En este territorio fundará varios asentamientos en cuatro años. Los cuales acabarán siendo destruidos a partir de una rebelión mapuche, iniciada en la batalla de Curalaba en 1598.

Otro argumento sobre la importancia de la Araucanía la encontramos en Garretón (1997:124) con el siguiente dato: La ciudad de la Imperial, fue fundada como "cabeza de reino". Pedro de Valdivia quería fundar la capital en este punto. Garretón además advierte otra idea interesante: es a partir de esta latitud donde la cordillera de los Andes se va haciendo más amable para atravesarla. Encontramos una idea similar en Cerda Hegerl (1995:5), aunque ella enfocándose más en la zona de Concepción. Ella señala que si no fuera por la guerra de Arauco y la defensa de los mapuches de su territorio probablemente esta habría sido la región más rica y poblada de la Capitanía General de Chile.

Probablemente esta era una zona muy poblada, así lo señala Bengoa (2008). Una zona así podría hacer pensar que se daban las condiciones para hacer de este lugar un lugar privilegiado. Ya era una política hispana el buscar lugares poblados, por lo general los lugares poblados ya hablan de lugares beneficiosos para el habitar. Además, disponen de bastante mano de obra para los conquistadores, que usaban a los indígenas para diferentes trabajos.

\subsection{Pedro de Valdivia y el modo de poblar}

Pedro de Valdivia es central para comprender el territorio de la Araucanía. Con los antecedentes revisados parece ser el primero quien pretende tener una visión para, de algún modo, construir y transformar el territorio. Por una parte, intenta fundar ciudades a ciertas distancias unas de otras y al mismo tiempo tener un punto de contacto hacia las pampas del oriente cruzando la cordillera de los Andes en la zona de la Araucanía.

Dentro de las características que buscaba para fundar una ciudad estaban la accesibilidad, la comunicación por mar y tierra; la cantidad de población aborigen; la riqueza material del lugar, sea oro o riqueza alimentaria.

Según Garretón (1997:118-119) en la estrategia usada por Valdivia se puede ver la intención de fundar ciudades bastante aisladas entre sí de manera de poder dominar mucho territorio en poco tiempo. Eso a la postre le traería problemas, según este mismo autor Valdivia demostraba con esta actitud un desconocimiento de la belicosidad de los indígenas pensando que estos aceptarían rápidamente el sometimiento español. Fruto de esta falta prudencia encuentra la muerte pocos años después de haber fundado las primeras ciudades españolas en la Araucanía. Murió a fines de 1553 después de ser aprisionado en un lugar llamado Tucapel.

La lista de asentamientos españoles que se construyeron al mando de Pedro de Valdivia fueron los siguientes: Concepción, 1550; La Imperial, 1551; Valdivia, 1552; Villarrica, 1552; Los Confines de Angol, 1553; Arauco, 1552, Tucapel, 1552; Purén, 1553. Algunos de estos (Arauco, Tucapel y Purén) no fueron asentamientos urbanos sino simples fuertes.

Hemos planteado un dibujo (figura 3 ) donde se ha seleccionado una curva de nivel a 200 metros de altura, y se han ubicado todos estos asentamientos hispanos sumando dos (Chillán y Yumbel) que se hicieron algunos años después de la muerte de Valdivia. Lo que se puede observar es la importancia de la cordillera de la costa. En ambos costados de esta cordillera se van fundando los puntos de avance español. Es interesante saber que todos estos puntos llegaron a convertirse en ciudades en el siglo XIX. La ubicación parece haber sido bien escogida. Solo un punto se observa a los pies de los Andes: la ciudad de Villarrica, insinuando este avance por los Andes hacia las pampas. Otro punto interesante para observar es que, gracias a la curva a $200 \mathrm{~m}$ podemos ver la que se repite un patrón: fundar asentamientos en tierras bajas, sumado a puntos de inflexión topográfica. Al menos entre las ciudades que se fundan en la época de P. de Valdivia ninguna se ubica en un gran llano, siempre buscan una "espalda". Un último apunte interesante es que si observamos la morfología que va dando la curva a $200 \mathrm{~m}$ vemos que un poco más al sur del río BíoBío se va formando un "embudo" territorial que se hace angosto hasta llegar a la zona conformada por los ríos Cautín y Toltén. De algún modo es esta dificultad topográfica cercana al río Bío-Bío la que, años más adelante, creará la frontera entre mapuches y la colonia española en Chile. 


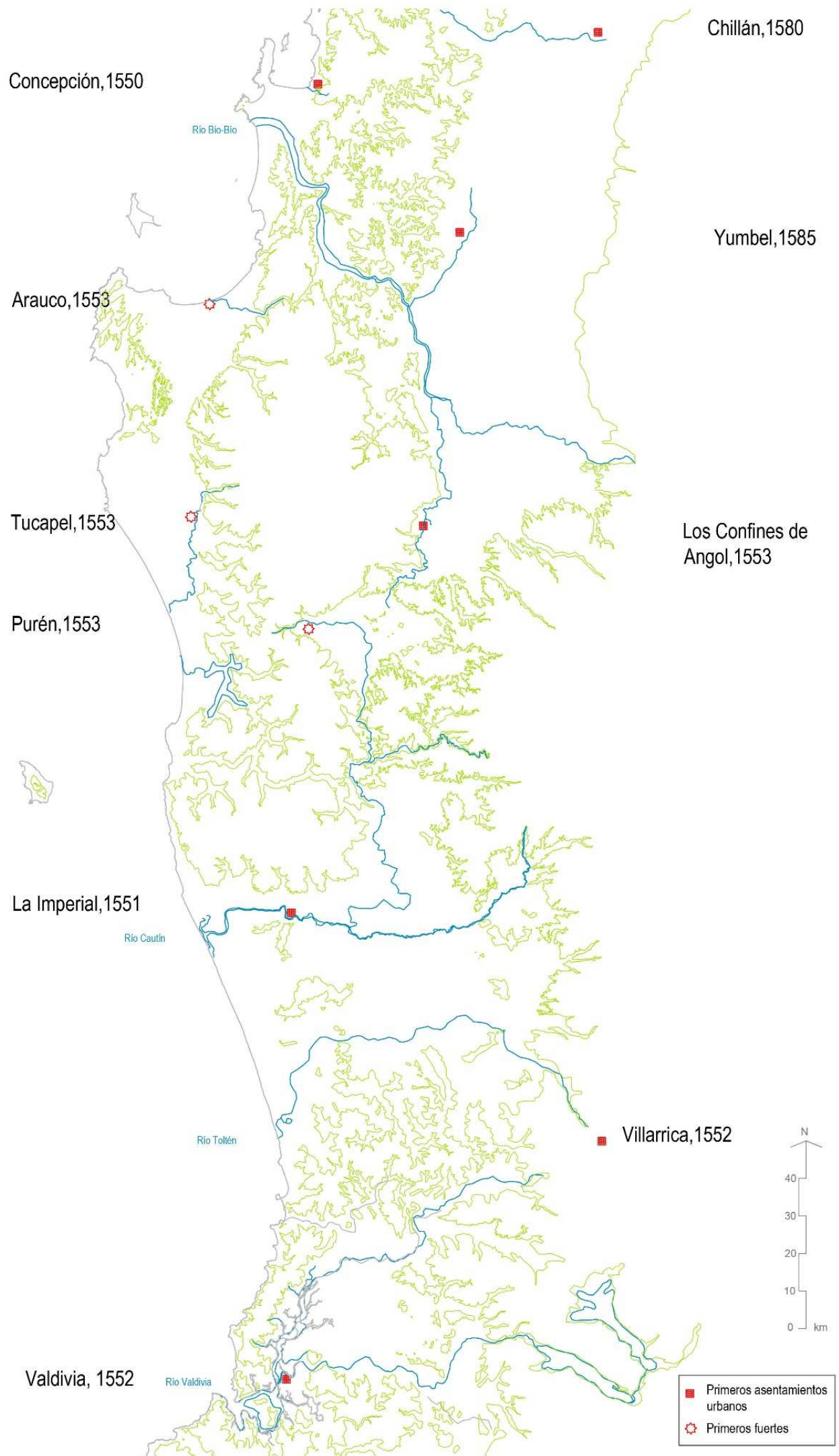

Figura 3. Asentamientos españoles, cursos de aguas y una curva de nivel a 200m. Fuente: Elaboración propia con base en cartografía IGM 


\subsection{La guerra y la destrucción de las ciudades. Nacimiento de una frontera. La línea del Bío-Bío}

A partir de la batalla de Curalaba en 1598 los mapuches destruyen las ciudades al sur del Bío Bío, y durante el siglo XVII habrá un continuo estado de guerra. A todo este período se le conoce como "La Guerra de Arauco", la cual detuvo la fundación de más asentamientos urbanos. Algunos autores señalan incluso que se inicia un proceso de ruralización de Chile. Todo este clima se tranquilizará hacia el siglo XVIII, donde habrá más estabilidad (Villalobos, 1995), y donde también se procederá a otra etapa de fundación de diversos pueblos en la zona del Chile central. A partir de la rebelión mapuche las fuerzas coloniales retroceden y se establece una frontera en el río Bío-Bío, y sobre esta frontera viene a establecerse una densidad de construcciones importante, sean nuevos asentamientos urbanos o nuevas fortificaciones militares.

La cantidad de fuertes fue especialmente alta en esta zona, así lo demuestra Guarda (1990:XIX) donde señala que en la zona de Concepción-Arauco habían 88 construcciones de este tipo y en Valdivia 57, en tanto en el resto del país el número era bastante menor.

En la figura 4 se ha dibujado, nuevamente el mismo encuadre pero con una curva de nivel a $400 \mathrm{~m}$, y solo con las construcciones territoriales hispanas del siglo XVIII, principalmente a partir de una cartografía hecha por Fray León Garavito en 1759 (Medina, 1952: s/n) y otras cartografías que aparecen en (Rosenblitt y Sanhueza, 2010:177-179) (Guarda, 1990:205) y los caminos que aparecen en una cartografía de Tomás López en 1777 (Gómez y Porto, 2006:431) Se ha intentado hacer coincidir todos los datos que contienen estas cartografías con la base actual del IGM.

Vemos en la figura 4 también la concentración de construcciones alrededor del río Bío Bío. Se han marcado en rojo las construcciones que estaban vigentes y en gris las que ya estaban destruidas. Es interesante ver un sistema de caminos más elaborados en los bordes y al norte del río Bío-Bío, mientras que, en el interior de la Araucanía, se reconocen solo dos caminos, de norte a sur, uno que pasa por la costa y otro por el valle central. También se observa una concentración de fuertes en la zona de Valdivia pero esta no llega hasta la cordillera como sí lo hace la zona del río Bío Bío, donde las construcciones han avanzado hacia lugares más altos, intentando "cerrar", de costa a montaña la Frontera.

El porqué de estas construcciones en esta zona tiene también explicaciones políticas. Existe consenso en que, entre mapuches y la colonia, se llevaron a cabo varios tratados o "parlamentos", y algunas fuentes señalan que el tratado de Quilín en 1641 vino a reconocer el límite entre la colonia y los mapuches en el río Bío Bío, reconociendo su autonomía (Grupo de Trabajo Legislación e Institucionalidad, 2009: 25).

\section{LA CONSTRUCCIÓN MAPUCHE SOBRE EL TERRITORIO}

Los mapuches no construyeron ciudades tal como lo hicieron otras culturas precolombinas. Sin embargo, tampoco se les cataloga como pueblos nómades recolectores. Lo que más se repite es que los antiguos mapuches fueron un pueblo trashumante, o algo que mediaba entre lo sedentario y lo nómade. Esto es de especial importancia. Una idea que podemos examinar en la literatura es que los mapuches, si bien no construyeron asentamientos urbanos, si construyeron asentamientos dispersos con un sentido de unidad. Estos se agrupan según diferentes grupos y varían en número y nombre según los autores. Para este trabajo, los niveles de agrupación mapuche que se han considerado son tres: El lof, el ayllarehue, y el vutanmapu. El lof es la agrupación básica de asentamiento mapuche, en tanto el ayllarrehue y el vutanmapu corresponden más bien a agrupaciones más grandes, de tipo político, para casos de guerra u otros asuntos colectivos. 


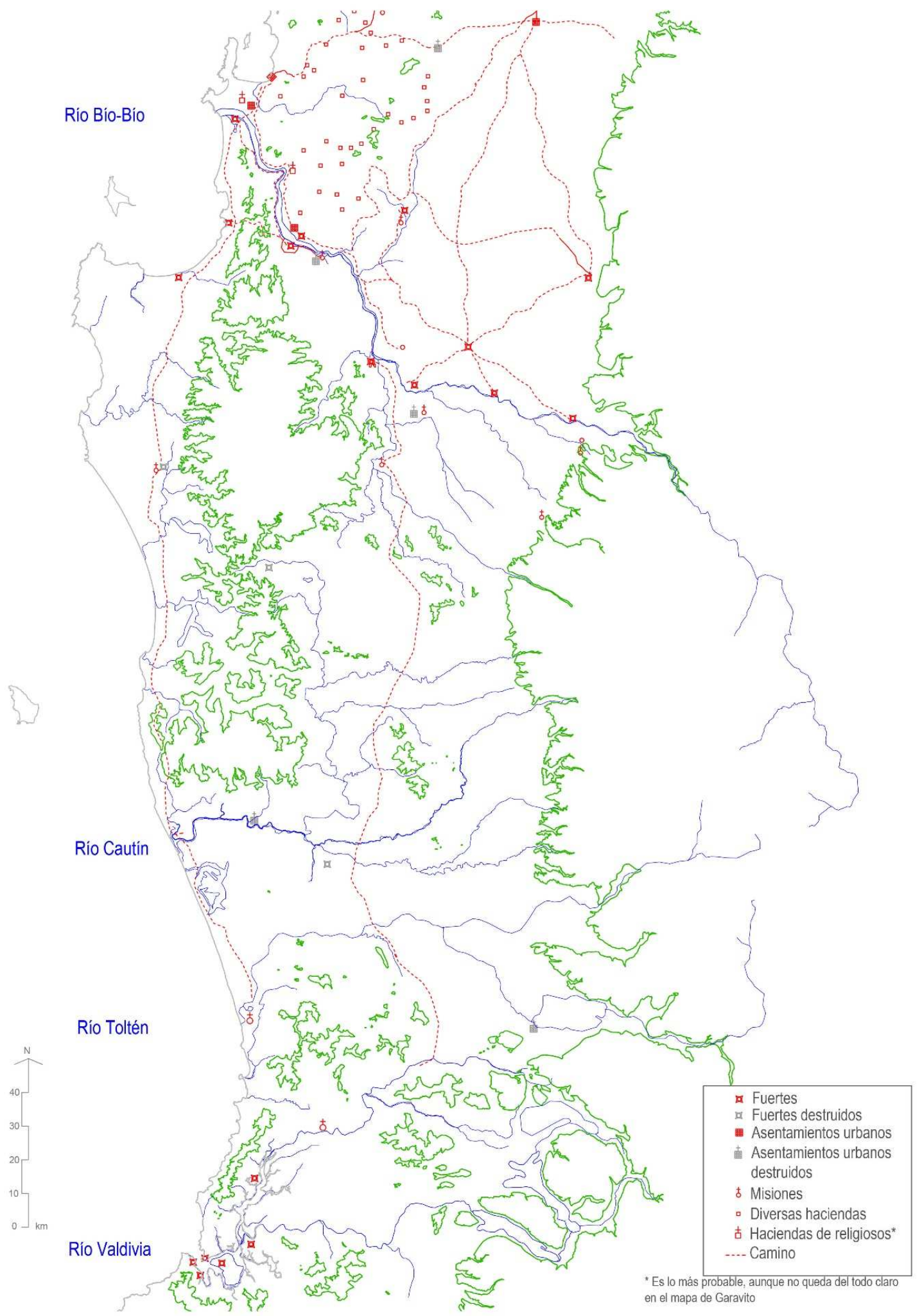

Figura 4. Asentamientos hispanos durante el siglo XVIII, con los ríos principales y una curva a 400m. Fuente: Elaboración propia a partir de cartografía IGM y cartografías aparecidas en Medina (1952, s/n), Rosenblitt y Sanhueza (2010:177-179), Gómez y Porto (2006:431) y Guarda (1990:205) 


\subsection{El lof, unidad básica de asentamiento}

El lof es la forma más básica de organización social según los estudios de historiadores y antropólogos. Estos, no tenían una forma establecida, pero eran agrupaciones sencillas con lazos familiares. La unidad básica del lof es la ruka, que viene a ser la vivienda mapuche. Intentar dibujar un lof no es fácil, no hay registros ni mapas fiables donde podamos establecer con seguridad la morfología de estos asentamientos. Lo que planteamos, en cuanto a su imagen, es más bien un esquema bastante básico (figura 5), donde el lof no viene a ser otra cosa que agrupaciones de puntos (rukas) donde en ellas hay un sentido de lo colectivo y las hace diferente de otros lofs.

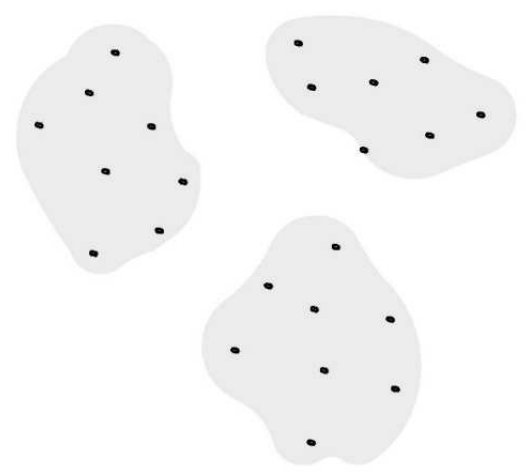

Figura 5. Esquema de unos lof. Fuente: Elaboración propia

Una buena síntesis para saber lo que es un lof, la encontramos en Bengoa (2008:159) señalando las siguientes características ${ }^{3}$ :

-está constituida por un "señor"

-tienen un lugar específico ("lugar señalado")

-se administra de la justicia

-es un lugar político de discusión.

En cuanto al número de población y estos asentamientos Vivar (citado en Bengoa 2008:157) dice que "...la población de un levo en 1558 fluctuaba entre 1.500 y 2.000 personas".

Después de diversos diálogos con estudiosos del tema planteamos que el lof no es otra que "la expresión de la familia mapuche en el territorio".

Aldunate (1996: 126-129) nos dice lo siguiente:

"El grupo local residencial, que antes recibía el nombre de lof y sus miembros lofche, está constituido por un patrón que parece haber sido siempre disperso, de acuerdo a descripciones de cronistas, aunque también hay algunas que destacan su nucleamiento (Núñez de Pineda 1863, 126). En él habitan en casas separadas y distantes los varones miembros del patrilinaje con sus mujeres e hijas solteras y las demás mujeres del patrilinaje que no han contraído matrimonio."

Con esta información inevitablemente podemos caer en la imagen de una típica "aldea" indígena: un grupo de casas juntas (sin necesariamente tener una forma estrictamente geométrica en su organización) organizadas sobre la base de algún tipo de un espacio común. Sin embargo Zavala (2011:64) escribe que el lof no es una aldea. Su opinión nos parece interesante para no caer en un formalismo apresurado respecto al asentamiento mapuche antes de la colonización chilena.

"El lof no constituye, sin embargo, una aldea, puesto que la proximidad geográfica de las casas que lo conforman no es suficiente para constituir una aglomeración. Fue justamente esta configuración espacial lo que planteó un enorme problema a la estrategia de dominación española basada principalmente en el ejercicio del control espacial de las comunidades indígenas" (Zavala, 2011:64)

\footnotetext{
${ }^{3}$ Bengoa se basa en Jerónimo de Vivar, quien, como otros autores o cronistas, no usa exactamente el término lof sino lebo o levo. Estas diferencias son frecuentes ya que el idioma mapuche, hasta donde sabemos, nunca fue escrito y cada autor interpreta los sonidos de forma diferente.
} 
Para nuestro interés el lof termina siendo una expresión de una ocupación dispersa en el territorio cuya expresión está vinculada a elementos naturales de la geografía y a la cosmovisión. De acuerdo a la cosmovisión mapuche, hay lugares señalados o especiales que no pueden ser ocupados ni para habitación ni para agricultura. Lo que pensamos es que su expresión corresponde al dibujo que hemos hecho (figura 5), este patrón de puntos dispersos pero anclados a un tipo de grupo particular, así mismo estos agrupados en un tronco familiar y algún punto ceremonial.

Aldunate (1996:117) nos da otra característica sobre el lof, lo define, durante la guerra de Arauco, como un asentamiento "móvil" que se mezclaba con una economía recolectora y horticultora. Según este autor también los mapuches en esta época aprenden el uso de animales como el caballo, ovejas y vacuno y esto afectará varios ángulos de la cultura. Esto sin duda genera un tipo diferente de sociedad comparada con aquella prehispánica, el uso y adaptación de animales como el caballo se ajusta mejor con este tipo de asentamiento más móvil.

Como ya se mencionó, no hay acuerdo sobre la terminología de los conceptos en cuanto a los asentamientos mapuches, en algunos casos al lof se le suele utilizar el mismo término que rehue. Zavala (2011:74), en este sentido, no encuentra una diferencia tan notoria entre lo que sería un lof y un rehue. Según él los "españoles del siglo XVIII se referían indistintamente con el término "parcialidad" tanto al lof como al rehue". (ibidem:74). Lo que sí nos dice es que tanto sea lof o rehue tal "parcialidad" descrita por los españoles se caracterizaba por dos factores: habitaciones que estaban próximas geográficamente y la presencia de un cacique o ulmen de cada "parcialidad". Latcham (1924:598) se atreve a hacer un cálculo de 470 rehues. A esta cantidad el mismo autor no le da una seguridad completa. Este dato, aunque no ciertamente exacto, nos ayuda a comprender poco a poco la naturaleza de este territorio y lo que se ha podido imaginar de él.

\subsection{Ayllarehues}

En lengua mapuche la palabra aylla significa "nueve". La conclusión más rápida nos dice que estos grupos estaban conformados por nueve rehues. Latcham (1924:597) deduce una cantidad de ayllarehues "a la llegada de los conquistadores españoles en el siglo XVI". Según este autor existían alrededor de 50 a 51 ayllarehues al sur del río Itata y al norte de la Isla de Chiloé.

También identifica un considerable número de ayllarehues con algunos de sus lebos que estaban incluidos dentro de cada uno, entre estos ayllarehues están: Purén, Cautén, Boroa, Rupücura, Maquehue, etc (ibidem.: 598-604).

Resulta importante señalar que la existencia de estas divisiones territoriales, al ser descubierta por los españoles, se les dió un uso que también jugaba al favor del control de este territorio. Esto lo encontramos a partir de Zavala (2011:74) quien nos dice que la identificación de estos ayllarehues sirvió para la organización tanto del ejército que quería conquistar como para los misioneros que querían evangelizar. Tanto es así que "los nombres de las misiones y fuertes retomaban aquellos de los ayllarehue, así, por ejemplo: Arauco, Boroa, Tucapel, Purén, Toltén". El mismo autor dice que esto fue "una forma de acomodamiento español a la realidad indígena".

Una diferencia entre los lofs (o rehues,) y los ayllarehues, es que, de acuerdo a la información que tenemos, los ayllarehues puede ser dibujados, al menos un dibujo con los límites aproximados de los ayllarehues descritos por Latcham (1924:598-602). Para construir el dibujo se han ubicado con un mapa actual los lugares que Latcham ha identificado. A partir de eso se intentan delimitar las áreas. El dibujo no pretende marcar con fuerza los "límites" o bordes, sino más bien, expresar áreas con limites difusos. Lo primero que se puede ver es que estas áreas corresponden a zonas bajas del territorio, parece obvio, pero esta observación es bastante útil para ir asentando los conceptos. Por otro lado, hasta ahora, en los trabajos hechos hacia el pueblo mapuche, son pocos los casos donde se han atrevido a dibujar o cartografiar el territorio mapuche. 


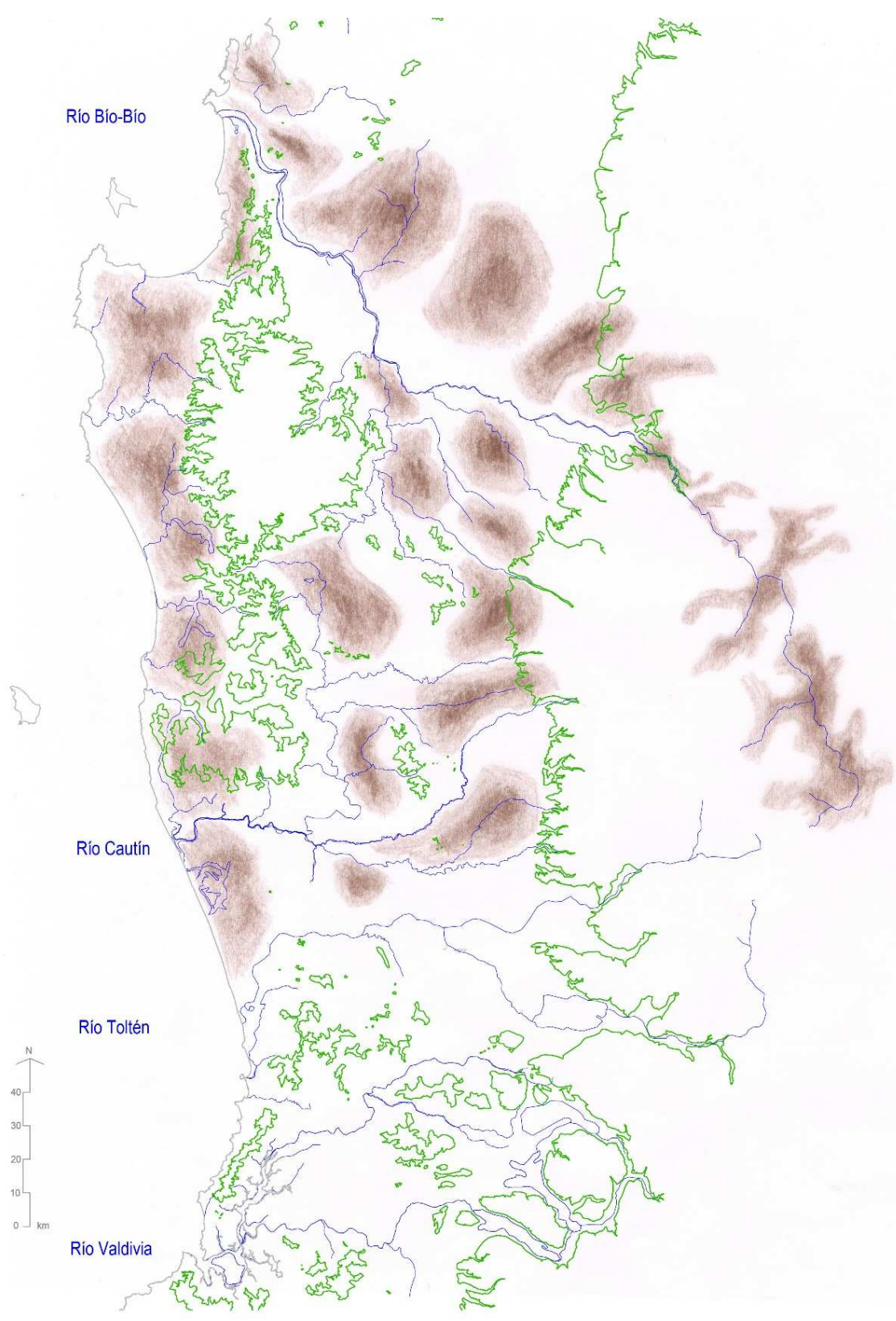

Figura 6. Diferentes ayllarehues según lo desprendido de Latcham.

Fuente: Elaboración propia con base en cartografía IGM y datos de Latcham (1924)

\subsection{Vutanmapus}

Hasta ahora no tenemos una definición única de lo que es un vutanmapu pero lo que sabemos con más certeza es que eran las organizaciones políticas mapuches de mayor tamaño, involucrando grandes áreas territoriales.

Según Zavala (2011:76) ${ }^{4}$ durante el siglo XVIII se reconoce, de parte de la colonia, la existencia de cuatro vutanmapus. Este mismo autor (2011:78 citando a Febres, 1765:674) presenta una enumeración hecha respecto a algunos ayllarewe que constituían los cuatro vutanmapu.

“-Lafquen-vutanmapu: Arauco, Tucapel, Imperial Baja, Boroa, Toltén Bajo.

-Lelfün-vutanmapu: Santa Juana, Angol, Repocura, Imperial Alta, Maquehua, Toltén Alto.

\footnotetext{
${ }^{4}$ Zavala usa como referencia esto: AGI, Ch 189, "Parlamento de Concepción celebrado por el presidente manuel de Salamanca el 13 de octubre de 1735".
} 
-Inapire-vutanmapu: Santa Fe, Nacimiento, Colhue, Chacaico, Quechereguas.

-Pire-vutanmapu: Santa Bárbara y "toda la cordillera de los Pehuenche"."

Zavala (2011:78-79) hace un par de aclaraciones respecto a estos vutanmapus. Por una parte, dice que estos que hemos mencionado no se encuentran al sur del rio Bío Bío, sino que incluyen a algunas facciones indígenas del norte de este río. Por otra parte, aclara que los vutanmapus no eran necesariamente agrupaciones para los casos de guerra, ya que en ocasiones estas alianzas podían darse entre distintos vutanmapus. Señala más bien que "eran conjuntos coherentes al interior de los cuales existían solidaridades, alianzas, circuitos de intercambios económicos y ceremoniales establecidos."

También encontramos otra definición de parte del cronista español Núñez de Pineda (él lo escribe utammapos):

"...son parcialidades de que se compone toda la tierra que habitan desde la costa hasta la cordillera, que se reparte en tres caminos que llaman rupus. La una parcialidad es de la costa, la otra la parte de la cordillera y la tercera de en medio; que cada una de estas parcialidades tiene su distrito conocido y su jurisdicción señalada." (Núñez de Pineda y Bascuñán, 1863:40-41)

En sintonía con esta última definición encontramos a Latcham (1924:139) quien nos da una descripción de solo tres vutanmapus, lo describe así:

"Estos tres distritos o fajas, que se extendían desde el Bío Bío hasta el Toltén, se llamaban respectivamente: Lavquén mapu o región tierra de la costa o del mar; Lelvún mapu-región o tierra de los llanos e Inapire mapu-región o tierra cerca de la nieve. Posteriormente se agregaron otros dos: Pire mapu región de la nieve o de la alta cordillera; y huilliche mapu tierra de la gente del sur." (Latcham, 1924:139)

De acuerdo a su descripción nos podemos imaginar más fácilmente (a diferencia de las descripciones de otros historiadores) la forma que podría tener estos tres vutanmapus en la Araucanía. Por el Norte limitando con el río Bío-Bío y por el sur con el río Toltén. Se observan las 3 franjas en sentido norte-sur las cuales serían los tres vutanmapus (figura 7).

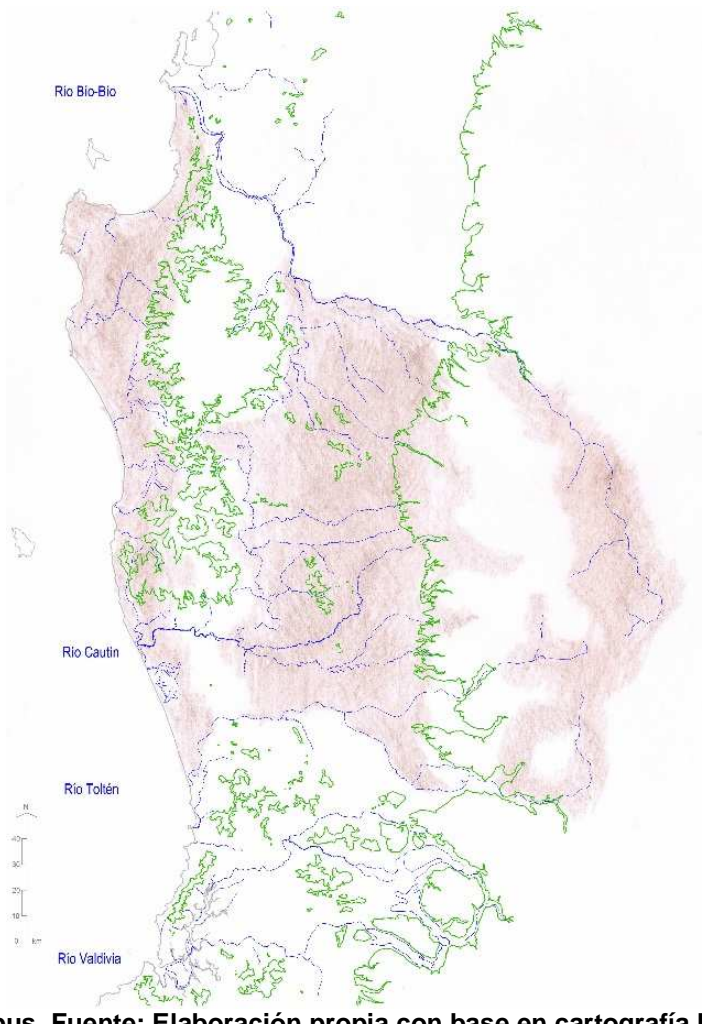

Figura 7. Tres vutanmapus. Fuente: Elaboración propia con base en cartografía IGM 


\subsection{Mapuches y la cartografía española}

En términos, quizás más concretos, la primera imagen sobre los asentamientos mapuches que hemos encontrado cartografiada corresponde a la cartografía de Garavito en 1759 (Medina, 1952:s/n) que hemos mencionado. La única señal que esta cartografía hace a las viviendas mapuches son unos simples triángulos agrupados alrededor de los ríos. De acuerdo a esto, hemos procedido a dibujar sobre una base cartográfica actual todos los puntos que dibuja Garavito (figura 8). Aquí podemos ver que los asentamientos mapuches tienen la tendencia a seguir los ríos. Esto concuerda de alguna manera con lo que ya ha planteado Bengoa (2008) señalando a los mapuches como "gente de los ríos".

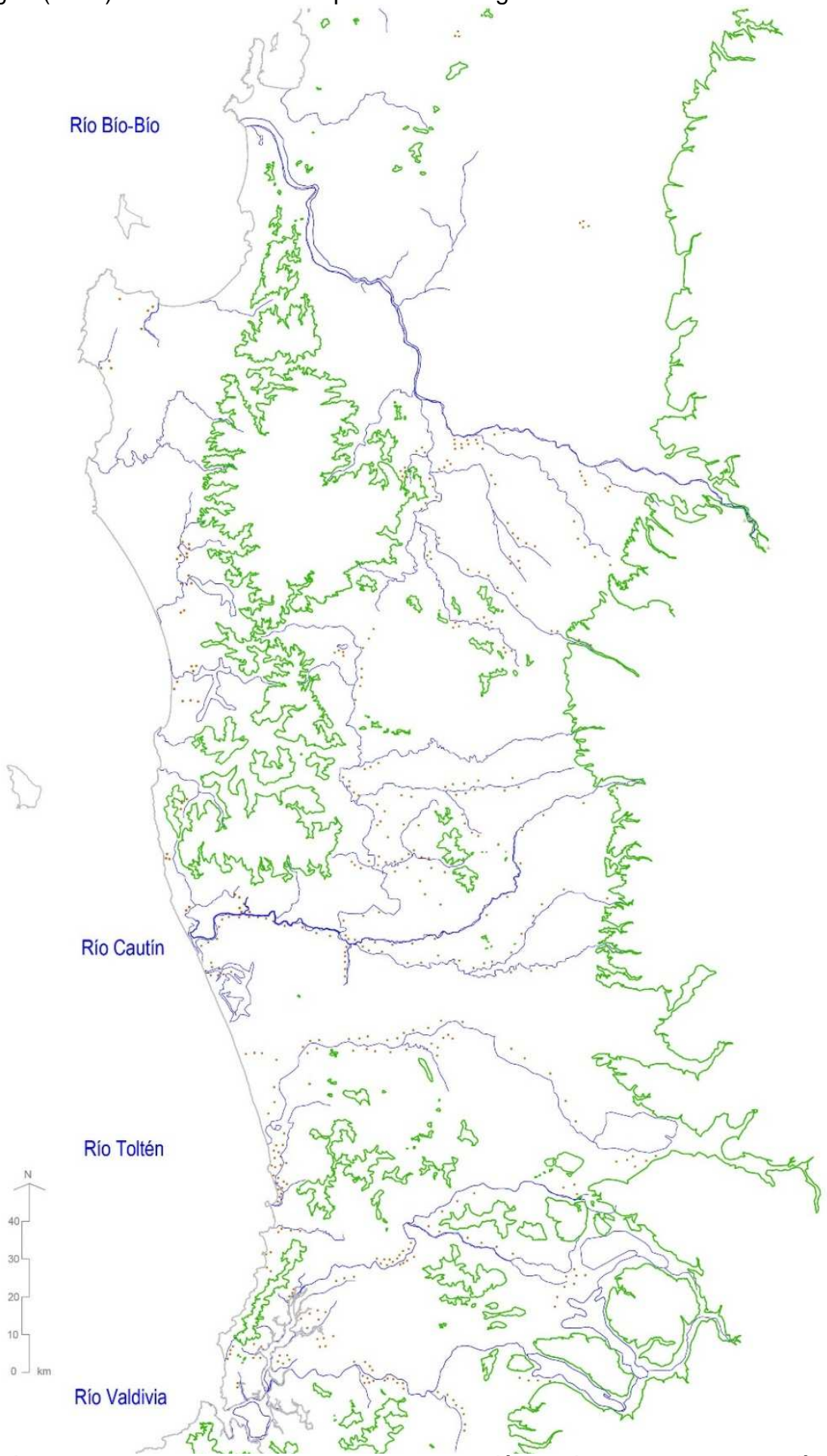

Figura 8. Asentamientos mapuches en el siglo XVIII. Fuente: Elaboración propia con base en cartografía IGM y Cartografía de Garavito (1759) 


\subsection{Caciques mapuches importantes en la República}

Conforme estudiamos los años más cercanos nos encontramos con más detalles. En cuanto a la población, por ejemplo, se calcula en un censo hecho a principios de siglo XIX que los habitantes de la Araucanía entre Bío Bío y Toltén eran unos 100 mil habitantes (Cruz, 2009: 354). Además, de esta misma época, se cuenta con buena información sobre grupos o facciones mapuches dentro del territorio, agrupaciones con caciques importantes (Guevara, 1902b:42), tanto de sus dominios territoriales, como de su poder y su relación con los otros (sean realistas, chilenos centralistas o chilenos "revolucionarios").

Guevara cita a los siguientes caciques (1902b, 36-37): Huenchunquir, Lincopi, Juan Cheuquemilla, caciques de la costa. Entre los del lado oriental de Nahuelbuta estaba Catrileo. Entre los del llano, entre los ríos Malleco hasta el Bureo y Mulchén estaba al mando Francisco Mariluan. También en el llano central, entre los ríos Traiguén y Cautín estaba la zona más densa de mapuches donde el principal cacique fue Mañil (o Mangin), a estos últimos grupos se les empezaba a conocer con el nombre de "arribanos". En Boroa, Truf Truf y Llaima estaban los caciques Calvuqueo y Curiqueo. Los grupos de la cordillera estaban bajo Martin Toriano.

También identifica a otros caciques que estuvieron al lado de los patriotas. Venancio Coñoepan, cacique del sur de Lumaco y Chol Chol. Juan Colipi en los territorios del actual pueblo de los Sauces y en los llanos de Angol hasta Temulemu, Quechereguas y Guadava. También menciona a un cacique llamado Melican, de la zona de los pehuenches.

Guevara (1902b) explica que ya existían facciones internas que hacían que unos y otros se aliaran o no con el gobierno chileno y da a Mañil una importancia superior dentro de los caciques de la época (1902b: 38-39) Guevara también se atreve a confeccionar el siguiente dibujo (figura 9) donde se observa un territorio mapuche con diversos tipos de grupos dentro de la Araucanía. Sin embargo, considerando los detalles que da sobre los caciques nos atrevemos también nosotros a hacer un dibujo (figura 10) con los dominios de los grandes caciques mapuches de siglo XX.

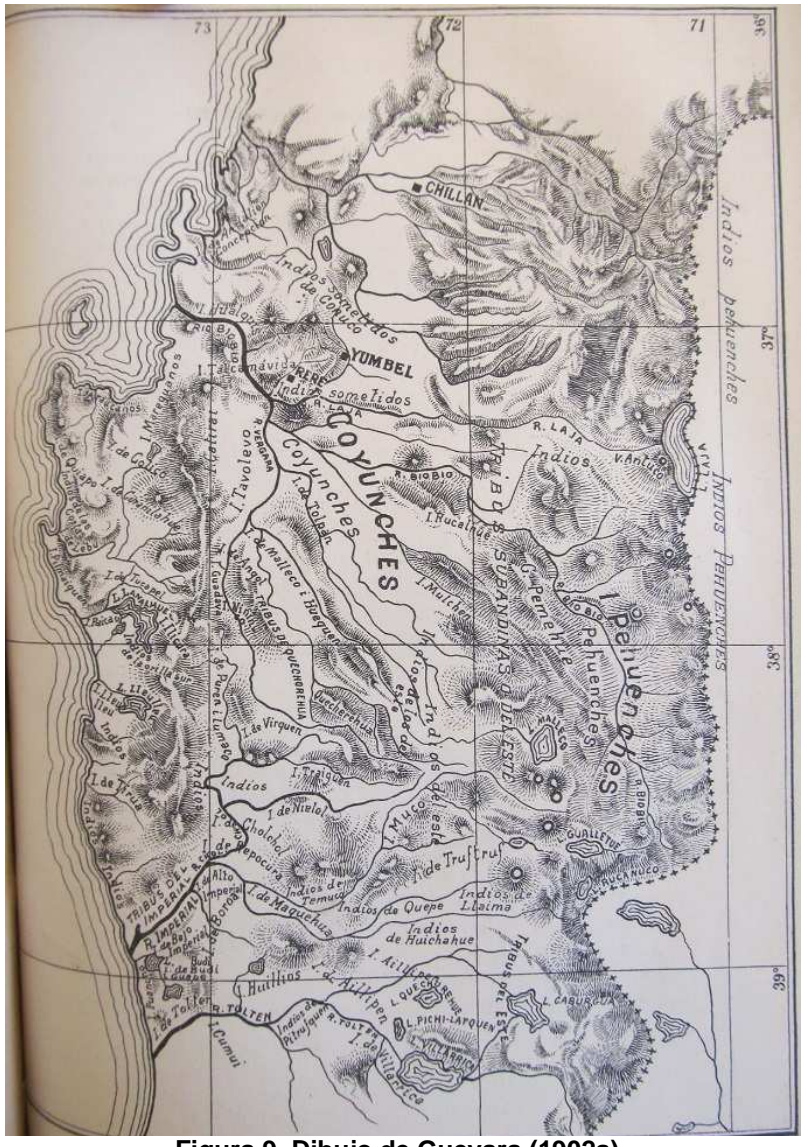

Figura 9. Dibujo de Guevara (1902a) 


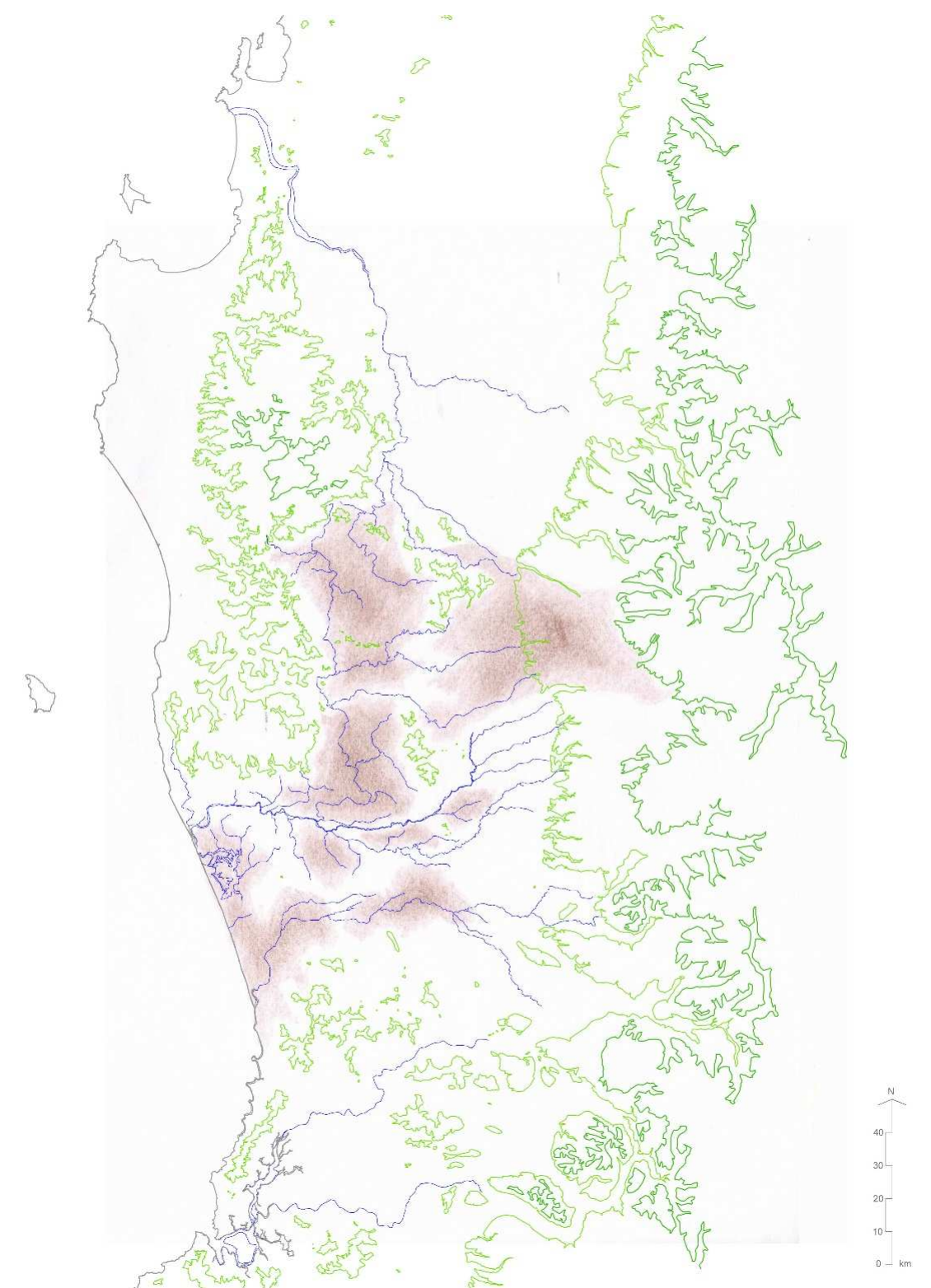

Figura 10. Ubicación de los dominios de los caciques del siglo XIX con curvas a 400 y 1000m. Fuente: Elaboración propia con base en cartografía IGM y datos en Guevara (1902b)

\section{LA REPÚBLICA Y LA MIRADA A LA ARAUCANÍA ANTES DE SU INVASIÓN}

Durante la época de la República la Araucanía fue un territorio con interrogantes respecto a su autonomía. Hubo debate, sin embargo, el hecho concreto es que, al menos físicamente, se había construido una densidad de construcciones importante en los alrededores del Bío-Bío, lo que en la práctica supone una frontera. En el año 1845 un viajero llamado Domeyco (2010) se internó en aquella Araucanía y publica una obra, y señala algunas características:

-Señala en su viaje que los lugares más poblados de la Araucanía eran los valles y ambos costados de la cordillera de la costa. (2010:20)

-Señala que la Araucanía poseía dos tipos de comunicación, una observación bastante obvia, pero nos ayuda a establecer certezas: un tipo de comunicación con el sentido norte-sur donde un camino pasa por la costa verticalmente y otro por el valle central, y sumado a eso una serie de caminos que recorren el territorio de oriente a poniente, atraviesan la cordillera de la costa para comunicar el valle central con la costa. (2010:20) 
Según Pinto (2010:xiii-xvi) Domeyco se encontró con una Araucanía diferente a la que había imaginado después de la lectura de la Araucana. Ya no existían aquellos antiguos guerreros, sino que este territorio poseía gente más bien laboriosa y con buenas relaciones hacia los hispanocriollos.

Domeyco también dibuja la Araucanía y en su cartografía se puede ver el encuadre que hemos venido discutiendo: el río Bío-Bío por el norte y el río Valdivia por el sur. Se ve una concentración todavía de construcciones alrededor del Bío-Bío y la Araucanía, prácticamente sin dibujos, salvo algunos asentamientos mapuches cercanos a la zona de la antigua La Imperial. Lo más preciso de este mapa son las zonas costeras y lo más difuso son los Andes. Lo interesante es que al menos será uno de los primeros mapas que algo hablará de la Araucanía, ya que, al menos en uno de los primeros mapas oficiales de la Geografía de Chile hecho por Claudio Gay aparece casi todo el país dibujado exceptuando la Araucanía, que aparece casi en blanco.

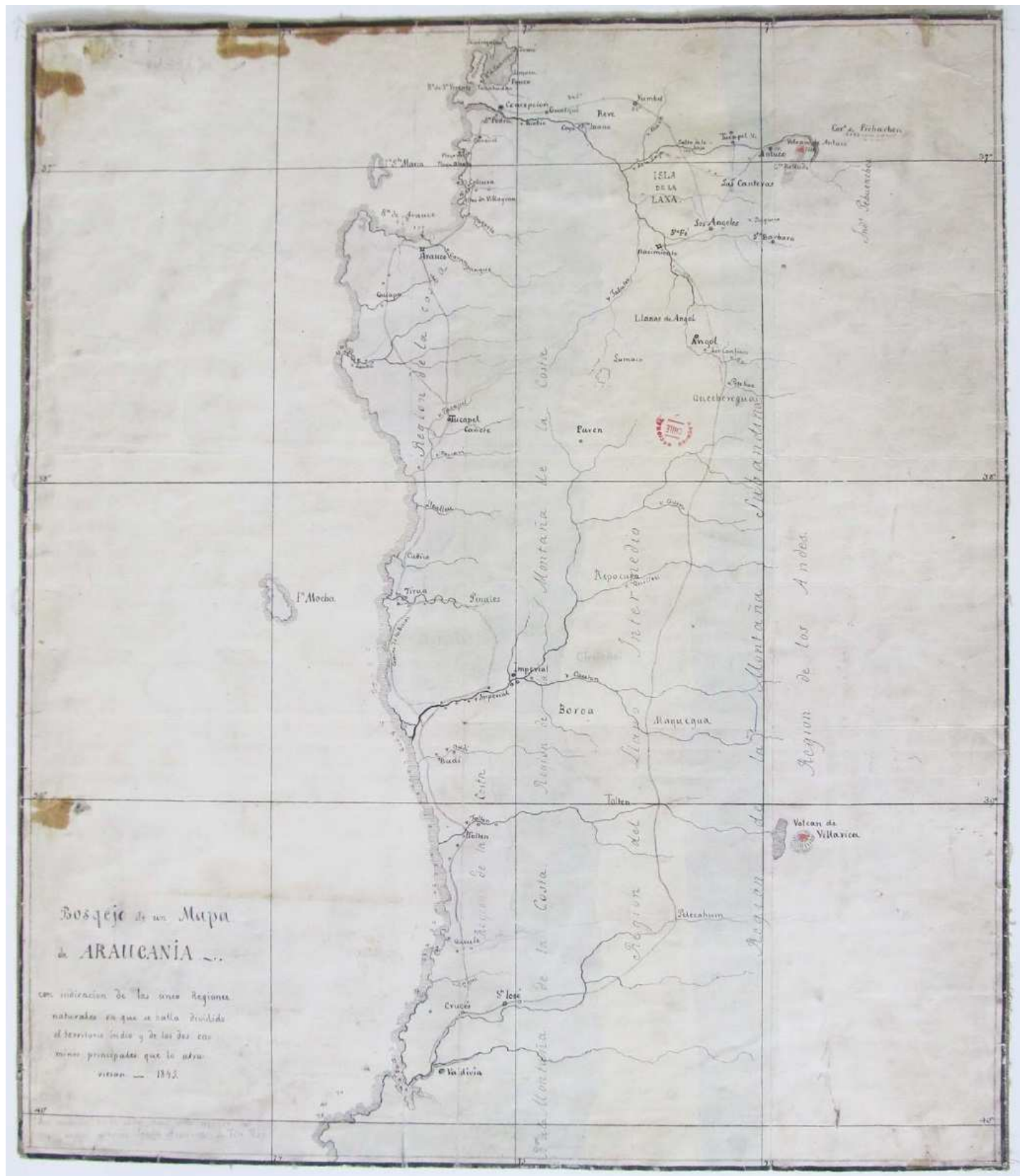

Figura 11. Mapa hecho por Domeyco en 1845. Fuente: Archivo Nacional de Chile 


\section{CONCLUSIONES}

El trabajo es un camino aún abierto. Por una parte, ha intentado buscar organizar toda aquella información dispersa de diversos autores cuando hablan de los hechos físicos y territoriales de la Araucanía, incluyendo el sentido de ello. Pero por otra, se ha intentado dibujar y empezar a postular hipótesis para entender este territorio. Si hay algo que este territorio aún no tiene es una expresión que lo haga entendible. Sabemos que su producción en cuanto a hechos territoriales no es mucha, pero útil para comprender no solo su historia sino sus voluntades territoriales, conocer sus capas, su palimpsesto.

En general vemos tres grandes patrones. En primer lugar, vemos la importancia de las tierras bajas tanto en la ocupación mapuche como en la colonial. Esto nos lleva a pensar que los Andes siempre fue una zona sin conquistar o desconocida. La ocupación española aparece como algo más puntual y la mapuche algo más dispersa y difusa. En segundo lugar, vemos la importancia de la cordillera de la costa a la hora de los asentamientos, especialmente en los asentamientos españoles, los cuales fueron ubicándose a ambos pies de esta cordillera. En tercer lugar, a través de los dibujos, observamos una Araucanía con dos límites, el río Bío-Bío y la zona de Valdivia, presentando una concentración de construcciones en dichas zonas, y dejando la conexión entre ellas, por tierra, solo a través de dos caminos norte-sur: el camino del valle interior y el camino de la costa.

Quizás la conclusión más general es que, a diferencia de lo que pudiera pensarse, cuando el Estado de Chile inicia su proceso de dominio en la Araucanía durante la segunda mitad del siglo XIX, no se encuentra con un territorio lleno de naturaleza y de incivilización total, sino con un territorio ya construido, con sus patrones territoriales (aunque sencillos) y que ya tenía de algún modo sus propias huellas. Los colonizadores chilenos se encuentran, por tanto, con una cultura territorial ya definida.

\section{BIBLIOGRAFÍA}

ALDUNATE, C. (1996). Mapuche: Gente de la Tierra. En J. HIDALGO, V. SCHIAPPACASSE, H. NIEMEYER, C. ALDUNATE, y P. MEGE (Eds.), Etnografía: Sociedades indígenas contemporáneas y su ideología. Santiago de Chile: Andrés Bello.

BENGOA, J. (2008). Historia de los antiguos mapuches del sur. Desde antes de la llegada de los españoles hasta las paces de Quilín. Siglos XVI y XVII (Tercera ed). Santiago de Chile: Catalonia Ltda.

CERDA-HEGERL, P. (1997). Fronteras del Sur. La región del Bio Bio y la Araucania chilena 1604 -1883. Temuco, Chile: Universidad de la Frontera.

COMISIÓN DE TRABAJO AUTÓNOMO MAPUCHE COTAM. (2009a). Primera parte del Informe Final de la Comisión de Trabajo Autónomo Mapuche. En Informe de la Comisión de Verdad Histórica y Nuevo Trato. Informes finales de los grupos de trabajo. Volumen 3 (pp. 567-1312). Santiago de Chile: Pehuén editores.

COMISIÓN DE TRABAJO AUTÓNOMO MAPUCHE COTAM. (2009b). Segunda parte del Informe Final de la Comisión de Trabajo Autónomo Mapuche. En Informe de la Comisión de Verdad Histórica y Nuevo Trato. Informes finales de los grupos de trabajo. Volumen 3 (pp. 1313-1914). Santiago de Chile: Pehuén editores. COÑA, P. (2006). Testimonio de un cacique. Santiago de Chile: Pehuén editores.

CRUZ, J. M. (2009). Memoria que a S. E. El Presidente de la República pasa el Señor General de División don José María de la Cruz, observando lo que en noviembre de 1861 presentó el señor coronel don Pedro Godoy, con motivo del pensamiento de realizar la ocupación del territorio... En SAAVEDRA, C, Documentos relativos a la ocupación de Arauco (pp. 351-369). Santiago de Chile: Cámara Chilena de la Construcción, Pontificia Universidad Católica de Chile, Dirección de Bibliotecas Archivos y Museos.

DILLEHAY, T. (1990). Araucanía: presente y pasado. Santiago de Chile: Andrés Bello.

DOMEYCO, I. (2010). La Araucanía y sus habitantes: Recuerdo de un viaje hecho en las provincias meridionales de Chile, en los meses de enero y febrero de 1845. Santiago de Chile: Cámara Chilena de la Construcción: Pontificia Universidad Católica de Chile: Dirección de Bibliotecas Archivos y Museos.

EIZAGUIRRE, X. (2001) La Construcción del territorio disperso: talleres de reflexión sobre la forma difusa. Barcelona: Edicions UPC

EIZAGUIRRE, X. (1985) Hipótesis de «entendimiento» territorial: sus elementos formales. Estudios territoriales. № 18, 181-196.

GARRETÓN, J. (1997). El urbanismo en Chile: Conquista y Colonia. Concepción, Chile: Ediciones Universidad de Concepción.

GREGOTTI, V. (1972) El Territorio de la arquitectura. Barcelona: G. Gili.

GRUPO DE TRABAJO LEGISLACIÓN E INSTITUCIONALIDAD. (2009). Informe del grupo de Trabajo Legislación e Institucionalidad. En Comisión de Verdad Histórica y Nuevo Trato (pp. 7-217). Santiago de Chile: Pehuén editores. 
GUARDA, G. (1978). Historia urbana del reino de Chile. Santiago de Chile: A. Bello.

GUARDA, G. (1990). Flandes indiano: las fortificaciones del reino de Chile 1541-1826. Santiago: Universidad Católica de Chile.

GUEVARA, T. (1902a). Historia de la civilización de Araucanía. Tomo II. Santiago de Chile: Imprenta, litografía i encuadernación Barcelona.

GUEVARA, T. (1902b). Historia de la civilización de Araucanía. Tomo III. Los Araucanos y la República. Santiago de Chile: Imprenta, litografía i encuadernación Barcelona.

LATCHAM, R. (1924). La organización social y las creencias religiosas de los antiguos araucanos. Santiago de Chile: Imprenta Cervantes.

LÓPEZ GÓMEZ, A., y MANSO PORTO, C. (2006). Cartografía del siglo XVIII: Tomás López en la Real Academia de la Historia. Madrid: Real Academia de la Historia, Departamento de Cartografía y Artes Gráficas.

MARIÑO DE LÓBERA, P., \& DE ESCOBAR, B. (1865). Crónica del reino de Chile. Santiago de Chile: Imprenta del ferrocarril.

MEDINA, J. T. (1952). Cartografía hispano colonial de Chile. Il atlas. Homenaje del Ejército de Chile a José T. Medina. Santiago de Chile: Instituto Geográfico Militar.

NUÑEZ DE PINEDA Y BASCUÑÁN, F. (1863). Colección de historiadores de Chile y de documentos relativos a la historia nacional. Tomo III. Cautiverio feliz, y razón de las guerras dilatadas de Chile. Santiago de Chile: Imprenta del ferrocarril.

PINTO RODRIGUEZ, J. (2010). Ignacio Domeyco. Viaje a la Araucanía en el año 1845 y otros documentos sobre la Frontera. In La Araucanía y sus habitantes: Recuerdo de un viaje hecho en las provincias meridionales de Chile, en los meses de enero y febrero de 1845 (pp. ix-Iviii). Santiago de Chile: Camara Chilena de la Construcción: Pontificia Universidad Católica de Chile: Dirección de Bibliotecas Archivos y Museos.

ROSENBLITT, J., y SANHUEZA, C. (2010). Cartografía histórica de Chile, 1778-1929. Biblioteca fundamentos de la construcción (Rosenblitt). Santiago de Chile: Versión Producciones Gráficas Ltda.

SOLÀ-MORALES, M. DE, y PARCERISA, J. (1981). La forma de un país. Quaderns d'Arquitectura I Urbanisme, Número ext.

VILLALOBOS, S. (1995). Vida fronteriza en la Araucanía: el mito de la Guerra de Arauco. Santiago de Chile: Andrés Bello.

ZAVALA, J. M. (2011). Los mapuches del siglo XVIII. Dinámica interétnica y estrategias de resistencia. Temuco, Chile: Universidad Católica de Temuco.

\section{CARTOGRAFÍA}

Instituto Geográfico Militar, IGM (Gentileza de la Corporación Nacional Forestal de Chile, CONAF)

Bosquejo de un mapa de Araucanía. Ignacio Domeyco,1845. 43,5 x51cm. Fuente: Archivo Nacional de Chile

\section{FUENTES ELECTRÓNICAS}

LABORATORIO DE URBANISMO DE BARCELONA (1881). La identitat del territori català. Les comarques, 1881 [en línea]. [Consultado el 9 de febrero de 2017] Disponible en: http://lub.upc.edu/web/arxiu_LUB/r_portada.html

EIZAGUIRRE, X. (1990) Las Componentes formales del territorio rural: los modelos de estructuras agrarias en el espacio metropolitano de Barcelona: la masía como modelo de colonización en Torelló [en línea]. Tesis doctoral. Escola Tècnica Superior d'Arquitectura de Barcelona, Universitat Politècnica de Catalunya. [Consultado el 25 de enero de 2017] Disponible en: http://www.tdx.cat/handle/10803/5874 